DOI: 10.1089/humc.2015.2520

\section{Advanced T-Cell Engineering for Cancer Therapy (ATECT)}

Contract No.: 602239; EU contribution: € 5,931,151; Total costs: $€ 7,780,342.20$; Starting date: 01/12/2013; Duration: 60 months

\section{Background and Objectives}

$\mathbf{T}$ -EFFECTOR CELLS (T-cells) are immune cells whose major role is surveillance for and destruction of virally infected cells. T-cells can be readily isolated from peripheral blood of patients or normal donors and cultured in vitro. By ex vivo manipulation, T-cells that recognize a specific cancer antigen can be selected and administered as a therapeutic. This therapy, known as adoptive immunotherapy, is an established method for treating a small group of human cancers, but is limited by an inability to generate T-cells specific for a wider range of cancer antigens.

More recently, gene therapy technology has provided a radically different strategy: by introducing a coding sequence for a chimeric antigen receptor (CAR) or a native T-cell receptor (TCR) using an integrating vector, we can rapidly confer any specificity to a large $\mathrm{T}$-cell population in vitro. We and others have tested such "positive" engineering in T-cells as a cancer therapeutic with clear and, in some cases, dramatic results.

Despite the considerable advance afforded us by genetic engineering, considerable barriers still exist, holding back the full potential of adoptive immunotherapy with engineered T-cells.

At present, a bespoke autologous therapeutic product must be produced for each patient. We believe that this limits practicality and scalability, restricting CARs to an experimental therapy in few specialized centers worldwide. Further, a lag time of weeks is needed for T-cell product generation. Production may fail or may be qualitatively different between patients. Recent success with B-cell malignancy represents the "low-hanging fruits" of CAR therapy. The problem of targeting other antigens, recruiting sufficient T-cells to sites of disease, and a hostile microenvironment are considerable barriers to function in broader range of human cancers, in particular solid tumors.

Recent genome engineering methods now give us an additional powerful T-cell engineering strategy to use alongside integrating vectors: Custom endonucleases targeting arbitrary sequences can result in inactivation of any desired genes"negative" gene therapy. The transcription activator-like effector nuclease (TALEN) technology is emerging as the most robust and effective technology platform for genomic engineering. We propose to develop advanced T-cell gene therapy with a combination of "positive" and "negative" engineering strategies, which we believe will revolutionize cellular therapy. Not only can we now engineer T-cells to have any specificity, but also we can render them resistant to hostile microenvironment and immunosuppressive agents and confer new properties. In addition, this combination of en- gineering strategies opens up the possibility of generating universal or "off-the-shelf" T-cells that will facilitate effective commercialization of T-cell therapy.

The core aim of this consortium is to test advanced T-cell therapy in early clinical studies and continue to develop it in the laboratory. We will first establish the feasibility and safety of the approach in a simple phase I study of TCR-edited, CD19 CAR donor T-cells in allogeneic hematopoietic stem cell transplantation. We will build on this study to a second phase I study, testing a more elaborate strategy to develop universal CAR T-cells that can be mass-produced and given "off the shelf." In addition, we will perform developmental and preclinical work, which we believe is necessary to expand the scope of engineered Tcells to more challenging malignancies by exploiting geneediting technology.

\section{Approach and Methodology}

We will implement clinical studies of CAR T-cells in patients with relapsed/refractory B-cell cancers. In addition to the use of lentiviral vectors to modify the T-cells, T-cell products will be subjected to genome editing to prevent allogeneic effects. In addition to this, more basic research and development will be applied to exploiting this concept of combining lentiviral engineering with genome editing for enhanced adoptive immunotherapy.

\section{Main Findings}

None reported yet.

\section{Expected Outcome}

Two complementary clinical studies of CD19-directed CAR therapy that together explore the potential and challenges for "off-the-shelf" CAR T-cell therapy. Development of new approaches to enhance adoptive immunotherapy by genome editing of $\mathrm{T}$-cell products.

\section{Major Publications}

None reported yet.

Coordinator: Prof. Martin Pule,m.pule@ucl.ac.uk

Partners: The ATECT consortium brings together 5 partners from 5 countries.

Martin Pule, Karl Peggs, Sergio Quezada, David Linch, and Adrian Thrasher, University College London, United Kingdom.

Andrew Scharenberg and Julianne Smith, Cellectis, France.

Dario Neri, Leonardo Giovannoni, and Chiara Falciani, Philogen, Italy.

Ton Schumacher and John Haanen, Nederlands Kanker Instituut, The Netherlands.

Burkhard Becher, Sonia Tugues, and Sabrina Hasler, Universität Zürich, Switzerland.

Website: atect-fp7.org

(c) European Union 2015. Published by Mary Ann Liebert, Inc. 